\title{
Emerging role of intestinal microbiota and microbial metabolites in metabolic control
}

\author{
Hilde Herrema $^{1} \cdot$ Richard G. IJzerman $^{2} \cdot$ Max Nieuwdorp $^{1,2,3}$
}

Received: 27 October 2016 / Accepted: 7 December 2016/Published online: 24 December 2016

(C) Springer-Verlag Berlin Heidelberg 2016

\begin{abstract}
The role of the intestinal microbiota and microbial metabolites in the maintenance of host health and development of metabolic disease has gained significant attention over the past decade. Mechanistic insight revealing causality, however, is scarce. Work by Ussar and co-workers demonstrates that a complex interaction between microbiota, host genetics and environmental factors is involved in metabolic disease development in mice. In addition, Perry and coworkers show that the microbial metabolite acetate augments insulin resistance in rats. These studies underscore an important role of the microbiota in the development of obesity and symptoms of type 2 diabetes in rodents. If causality can be demonstrated in humans, development of novel diagnostic and therapeutic tools that target the gut microbiota will have high potential.
\end{abstract}

Keywords Gut microbiota $\cdot$ Insulin resistance $\cdot$ Obesity . Short-chain fatty acids $\cdot$ Type 2 diabetes

\section{Abbreviations \\ BBB Blood-brain barrier \\ FMT Faecal microbiota transfer}

Hilde Herrema

h.j.herrema@amc.uva.nl

1 Department of Experimental Vascular Medicine, Academic Medical Center, University of Amsterdam, Meibergdreef 9, 1055

AZ Amsterdam, the Netherlands

2 Department of Internal Medicine, Free University Medical Center, Amsterdam, the Netherlands

3 Wallenberg Laboratory, University of Gothenburg, Gothenburg, Sweden
GSIS Glucose-stimulated insulin secretion

HFD High-fat diet

ICV Intracerebroventricular

SCFA Short-chain fatty acid

The symbiotic relationship with our intestinal microbiota is exemplified by a broad range of beneficial effects for the host metabolism, including digestion of complex carbohydrates and maturation of the immune response. In addition, the intestinal microbiota serves an endocrine role by producing a vast amount of metabolites with the potential to modulate host metabolism [1]. The intestinal microbiota has, as such, also been implicated in the development of obesity and type 2 diabetes in human and rodent models [2]. This adds another layer of complexity to studying the origin of these multifactorial metabolic pathologies that arise from a complicated interplay between genetic risk factors and external factors, including dietary intake and physical activity. This commentary discusses two recent rodent studies on the potential importance of the intestinal microbiota in metabolic disease. Ussar and co-workers [3] studied the complex interaction between environmental and genetic factors in microbiota-related metabolic disease development in mice, while Perry and co-workers [4] investigated a potential mechanism underlying microbiota-driven disease development using a rat model.

Colonisation of the gastrointestinal tract starts as early as in utero [5], and a core microbiome composed mainly of Bacteroidetes, Firmicutes, Actinobacteria, Proteobacteria and Verrucomicrobia is established around the third year of life. Extrinsic factors, including host genetic makeup [6], cohousing/family members [7], oral antibiotic use early in life [8] and diet [9] are dominating factors in shaping the intestinal microbiota. Whether these early life events are determinants of microbiota-related disease in later life remains to be 
established. Prospective large cohort studies addressing these factors in relation to microbiota composition will provide important insight into the role of the intestinal microbiota in the (patho)physiology of disease $[10,11]$.

A reduced number of bacterial genes (bacterial richness) in the intestine with a concomitant increase in inflammatory profile $[12,13]$ and increased circulating levels of bacterial $16 \mathrm{~s}$ rDNA [14] have been suggested to have predictive value for the development of obesity-associated insulin resistance. Nevertheless, causality cannot be proven in observational studies and, unfortunately, experimental human data are extremely limited. Interventions with significant impact on intestinal microbiota composition such as faecal microbiota transfer (FMT) and antibiotic treatment have thus far shown mild [15] or no clinically relevant effect [16], respectively, on host metabolic health. Data implicating a causal role for the intestinal microbiota and/or their metabolites in the development of metabolic disease mainly originate from studies in rodent models. Colonisation of germ-free mice, which lack intestinal microbiota, with microbiota from obese murine or human donors, causes them to gain more weight compared with counterparts who receive a lean transplant $[17,18]$. Other interventions that significantly affect murine intestinal microbiota composition with causal consequence for the development of obesity and related phenotypes include cohousing [19, 20], gastric bypass [21] and antibiotic treatment [22].

\section{Environment, host genetics and diet affect microbiota composition and its effect on host metabolism}

The complexity and importance of factors that interact to determine intestinal microbiota composition and metabolic disease development was particularly illustrated in work from Ussar and co-workers [3]. The researchers assessed the longterm effects of environmental changes on the interaction between host genetics, microbiota composition and metabolic phenotype in three commonly used mouse models with differing susceptibility to high-fat diet (HFD)-induced obesity and diabetes. Analysis of the faecal microbiota of the obesity/diabetes-prone C57Bl/6J (B6J) and obesity/diabetesresistant 129S1/SvImJ (129J) mouse from The Jackson Laboratory and the obesity-prone but diabetes-resistant 129S6/SvEvTac (129T) mouse from Taconic showed that diet, strain and birthplace were the strongest determinants of microbiota composition in all groups. These mouse models were subsequently maintained in the same facility for three to six generations. Interestingly, the phenotypes of the genetically-related $129 \mathrm{~T}$ and $129 \mathrm{~J}$ mice, which differ in terms of obesity development when obtained from separate facilities, resembled each other when maintained in the same facility for several generations. The changes in the metabolic phenotype could be associated with changes in specific bacterial taxa. Although facility-specific bacterial abundance increased in all models over time, the phenotype of the B6J strain remained fairly unaffected [3].

When germ-free mice received FMT from vendor-born B6J, $129 \mathrm{~J}$ and $129 \mathrm{~T}$ mice maintained on an HFD, mice that received a B6J or 129T transplant exhibited reduced glucose homeostasis. Mice receiving a 129J transplant had an ameliorated response to diet-related glucose-derangements. Although there were no differences in weight gain between any of the recipient groups, these data indicate that vendor-based intestinal microbiota can transfer part of the glucose metabolic phenotype. Additionally, observations that genetically identical mice (the 129 models) have different responses to HFD feeding when raised in different facilities negatively impacts controllability and reproducibility of metabolic studies in rodents [3]. Although data from the studies performed by Ussar and coworkers clearly suggest that crosstalk between microbiota, host genetic background and dietary challenge plays a significant role in development of metabolic disease, mechanisms underlying these observations still need to be determined.

\section{Role of specific bacterial metabolites in regulation of host metabolism}

Mechanistic insight into aspects of microbiota-mediated regulation of the host metabolism is crucial for development of strategies to treat obesity and related disease at the intestinal level. The vast number of yet-to-be-explored bacterial metabolites are likely to have a significant impact on human physiology and disease development. Their mechanism of action is probably not restricted to the (local) intestinal environment but extents to peripheral tissues [23]. The use of multi-omics approaches will reveal crucial insight into systemic effects of the intestinal microbiota on host metabolism, although the interpretation of causality/relevance will be highly challenging. The use of dedicated animal models [24] can, however, shed light on the effect of bacterial metabolites on host metabolic control.

Short-chain fatty acids (SCFAs), products of fermentation of non-digestible fibres by colonic microbes, are currently the most studied bacterial products because of their suggested beneficial health effects. A diet rich in non-digestible fibres has been associated with reduced risk of obesity [25], whereas the intestinal microbiota of diabetic individuals is relatively depleted in SCFA-producing bacterial species [26, 27]. In mice, dietary supplementation of the SCFA butyrate has been shown to prevent weight gain and improve insulin sensitivity by increasing energy expenditure [28]. In line with this, acetate, propionate and butyrate could prevent HFD-induced obesity and insulin resistance by causing a shift from lipogenesis to fatty acid oxidation in liver and adipose tissue [29]. 
Butyrate and propionate have been shown to induce intestinal gluconeogenesis, which, through an intestine--brain neural circuit, improves peripheral glucose production and insulin sensitivity [30].

Perry and co-workers recently showed that the SCFA acetate acts on parasympathetic activity to increase food intake and promote glucose-stimulated insulin secretion (GSIS) in a rat model [4]. Interestingly, acetate turnover, but not that of butyrate or propionate, was found to be increased in the colon, caecum and brain of rats fed an HFD compared with chow-fed rats. Since most rodent laboratory HFDs are basically devoid of dietary fibre, the source of microbiota-derived acetate remains somewhat illusive.

Infusion of acetate, but not butyrate or propionate, in chowfed rats mimicked increased GSIS as observed in HFD-fed rats suggesting that circulating acetate levels drive increased GSIS [4]. In line with this, FMT from HFD-fed rats to chow-fed controls increased acetate turnover and GSIS in the recipient rat. Moreover, germ-free mice that received a transplant from HFD-fed donors had a twofold increase in acetate production compared with recipients of a transplant from a chow-fed donor.

Interestingly, intracerebroventricular (ICV) and nucleus tractus solitarius injection of acetate increased GSIS without increasing circulating acetate levels. The ICV effect on GSIS could be blocked by methylatropine, a parasympathetic blocker that does not pass the blood-brain barrier (BBB). Finally, chronic intragastric administration of acetate in chow-fed rats replicated HFD-induced metabolic derangements, including increased food intake and GSIS, which could be prevented by vagotomy. Nevertheless, acetate-infused rats had significant weight gain, which could be an important confounding factor for metabolic derangements [3].

This study indicates that acetate acts centrally, specifically in the parasympathetic nervous system, to increase food intake, GSIS and fat deposition. Although a causal role for a microbiota-derived metabolite in the development of insulin resistance is shown, data are in sharp contrast with studies performed in HFD-fed mice. In the latter model, intravenous and colonic administration of acetate reduced food intake and weight gain by activating anorectic signalling pathways in the hypothalamic region of the brain [31]. It remains to be determined whether the contradictory effects of acetate administration in rats and mice is due to a species-specific response to acetate. In addition, it would be interesting to supplement the diets of rats with SCFAs to assess how the effect relates to effects observed in mice [28, 29]. In humans, acetate has also been shown to cross the BBB [32], and has been associated with increased short-term subjective satiety ratings [33] and reduction in body weight [34]. Direct (and indirect) roles of SCFAs in metabolic health in humans are therefore largely unknown. Ongoing studies with both oral and rectal supplementation of SCFAs in humans might soon provide more insight.

\section{Future perspectives}

The studies performed by Ussar and Perry and their colleagues suggest that the intestinal microbiota is an important

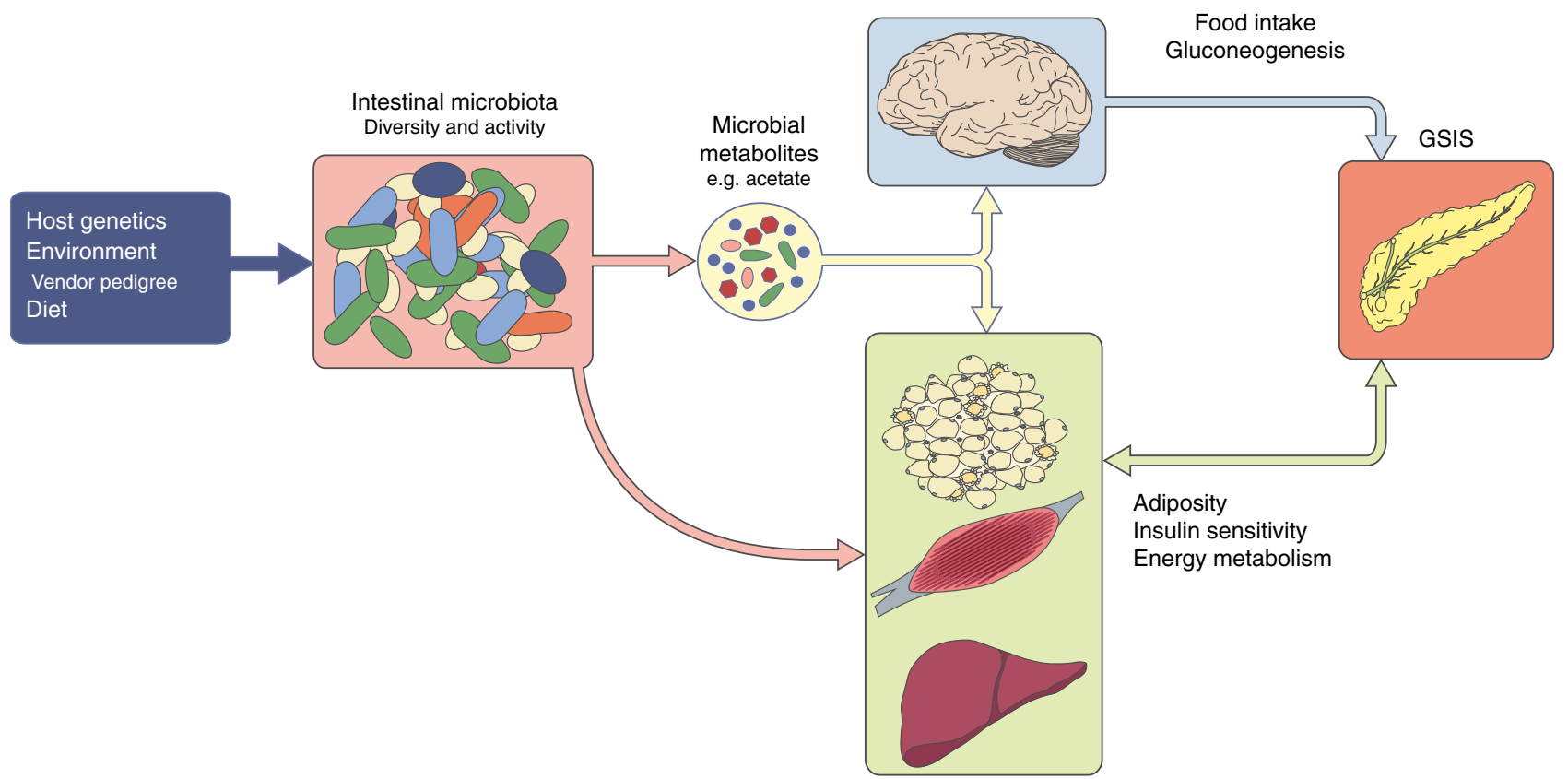

Fig. 1 Host genetics, diet and environment (including vendor pedigree) interact to determine intestinal microbiota composition and metabolic disease development. The vast number of microbial metabolites, including acetate, has a significant impact on host metabolism. The microbial secretome might therefore hold important new clues to unravel the role of the intestinal microbiota in the development of obesity and type 2 diabetes 
participant in rodent metabolism (Fig. 1). Although these studies underscore a role for the microbiota in the development of obesity and type 2 diabetes, studies showing causal effects in rodents should be confirmed in humans. If results on causality can be confirmed in humans, further research on human intestinal microbiota may lead to the development of novel diagnostic and therapeutic tools. Challenges in attempting to determine causality in humans include biases in associations between altered microbiota composition and development of human disease. Such associations are generally based on the accessible microbial pool in the faecal compartment. The composition and potential role of the microbiota community in other parts of the gastrointestinal tract is unexplored, as are implications thereof for host metabolic health (e.g. differential metabolic effects of microbial-produced SCFA in either the proximal or distal intestine) [15]. Furthermore, in many studies information on important drivers of microbiota composition, such as dietary habits and (history of) medication (such as antibiotics) is lacking. Despite these challenges, identification of specific strains or 'tailor-made' bacterial mixtures while improving engraftment [35] might provide interesting opportunities for the study and treatment of human metabolic disease.

Funding HH is supported by CVON 2012 grant (IN-CONTROL) (2012-03) to MN. MN is supported by a ZONMW-VIDI grant 2013 (016.146.327).

Duality of interest MN is on the advisory board of Caelus Health and Seres Therapeuticals and has received lecture fees from AstraZeneca, EliLilly and MSD.

Contribution statement $\mathrm{HH}$, RGIJ and MN were responsible for drafting the commentary and for critically revising it. All authors have approved the published version.

\section{References}

1. Wikoff WR, Anfora AT, Liu J et al (2009) Metabolomics analysis reveals large effects of gut microflora on mammalian blood metabolites. Proc Natl Acad Sci U S A 106:3698-3703

2. Khan MT, Nieuwdorp M, Backhed F (2014) Microbial modulation of insulin sensitivity. Cell Metab 20:753-760

3. Ussar S, Griffin NW, Bezy O et al (2015) Interactions between gut microbiota, host genetics and diet modulate the predisposition to obesity and metabolic syndrome. Cell Metab 22:516-530

4. Perry RJ, Peng L, Barry NA et al (2016) Acetate mediates a microbiome-brain- $\beta$-cell axis to promote metabolic syndrome. Nature 534:213-217

5. Jimenez E, Marin ML, Martin R et al (2008) Is meconium from healthy newborns actually sterile? Res Microbiol 159:187-193

6. Goodrich JK, Waters JL, Poole AC et al (2014) Human genetics shape the gut microbiome. Cell 159:789-799
7. Faith JJ, Colombel JF, Gordon JI (2015) Identifying strains that contribute to complex diseases through the study of microbial inheritance. Proc Natl Acad Sci U S A 112:633-640

8. Tamburini S, Shen N, Wu HC, Clemente JC (2016) The microbiome in early life: implications for health outcomes. Nat Med 22:713-722

9. Charbonneau MR, Blanton LV, DiGiulio DB et al (2016) A microbial perspective of human developmental biology. Nature 535:48-55

10. Bonder MJ, Kurilshikov A, Tigchelaar EF et al (2016) The effect of host genetics on the gut microbiome. Nat Genet 48:1407-1412

11. Zhernakova A, Kurilshikov A, Bonder MJ et al (2016) Populationbased metagenomics analysis reveals markers for gut microbiome composition and diversity. Science 352:565-569

12. Cotillard A, Kennedy SP, Kong LC et al (2013) Dietary intervention impact on gut microbial gene richness. Nature 500:585-588

13. Le Chatelier E, Nielsen T, Qin J et al (2013) Richness of human gut microbiome correlates with metabolic markers. Nature 500:541-546

14. Amar J, Serino M, Lange C et al (2011) Involvement of tissue bacteria in the onset of diabetes in humans: evidence for a concept. Diabetologia 54:3055-3061

15. Vrieze A, Van Nood E, Holleman F et al (2012) Transfer of intestinal microbiota from lean donors increases insulin sensitivity in individuals with metabolic syndrome. Gastroenterology 143:913916, e917

16. Reijnders D, Goossens GH, Hermes GD et al (2016) Effects of gut microbiota manipulation by antibiotics on host metabolism in obese humans: a randomized double-blind placebo-controlled trial. Cell Metab 24:63-74

17. Ridaura VK, Faith JJ, Rey FE et al (2013) Gut microbiota from twins discordant for obesity modulate metabolism in mice. Science 341:1241214

18. Turnbaugh PJ, Ley RE, Mahowald MA, Magrini V, Mardis ER, Gordon JI (2006) An obesity-associated gut microbiome with increased capacity for energy harvest. Nature 444:1027-1031

19. Henao-Mejia J, Elinav E, Jin C et al (2012) Inflammasomemediated dysbiosis regulates progression of NAFLD and obesity. Nature 482:179-185

20. Upadhyay V, Poroyko V, Kim TJ et al (2012) Lymphotoxin regulates commensal responses to enable diet-induced obesity. Nat Immunol 13:947-953

21. Liou AP, Paziuk M, Luevano JM Jr, Machineni S, Turnbaugh PJ, Kaplan LM (2013) Conserved shifts in the gut microbiota due to gastric bypass reduce host weight and adiposity. Sci Transl Med 5: 178ra141

22. Cox LM, Yamanishi S, Sohn J et al (2014) Altering the intestinal microbiota during a critical developmental window has lasting metabolic consequences. Cell 158:705-721

23. Schroeder BO, Backhed F (2016) Signals from the gut microbiota to distant organs in physiology and disease. Nat Med 22:10791089

24. Ussar S, Fujisaka S, Kahn CR (2016) Interactions between host genetics and gut microbiome in diabetes and metabolic syndrome. Mol Metab 5:795-803

25. Cho SS, Qi L, Fahey GC Jr, Klurfeld DM (2013) Consumption of cereal fiber, mixtures of whole grains and bran, and whole grains and risk reduction in type 2 diabetes, obesity, and cardiovascular disease. Am J Clin Nutr 98:594-619

26. Karlsson FH, Tremaroli V, Nookaew I et al (2013) Gut metagenome in European women with normal, impaired and diabetic glucose control. Nature 498:99-103

27. Qin J, Li Y, Cai Z et al (2012) A metagenome-wide association study of gut microbiota in type 2 diabetes. Nature 490:55-60

28. Gao Z, Yin J, Zhang J et al (2009) Butyrate improves insulin sensitivity and increases energy expenditure in mice. Diabetes 58 : 1509-1517 
29. den Besten G, Bleeker A, Gerding A et al (2015) Short-chain fatty acids protect against high-fat diet-induced obesity via a PPAR $\gamma$ dependent switch from lipogenesis to fat oxidation. Diabetes 64: 2398-2408

30. De Vadder F, Kovatcheva-Datchary P, Goncalves D et al (2014) Microbiota-generated metabolites promote metabolic benefits via gut-brain neural circuits. Cell 156:84-96

31. Frost G, Sleeth ML, Sahuri-Arisoylu M et al (2014) The short-chain fatty acid acetate reduces appetite via a central homeostatic mechanism. Nat Commun 5:3611

32. Jiang L, Gulanski BI, De Feyter HM et al (2013) Increased brain uptake and oxidation of acetate in heavy drinkers. J Clin Invest 123: $1605-1614$
33. Ostman E, Granfeldt Y, Persson L, Bjorck I (2005) Vinegar supplementation lowers glucose and insulin responses and increases satiety after a bread meal in healthy subjects. Eur J Clin Nutr 59:983-988

34. Kondo T, Kishi M, Fushimi T, Ugajin S, Kaga T (2009) Vinegar intake reduces body weight, body fat mass, and serum triglyceride levels in obese Japanese subjects. Biosci Biotechnol Biochem 73:1837-1843

35. Maldonado-Gomez MX, Martinez I, Bottacini F et al (2016) Stable engraftment of Bifidobacterium longum AH1206 in the human gut depends on individualized features of the resident microbiome. Cell Host Microbe 20:515-526 